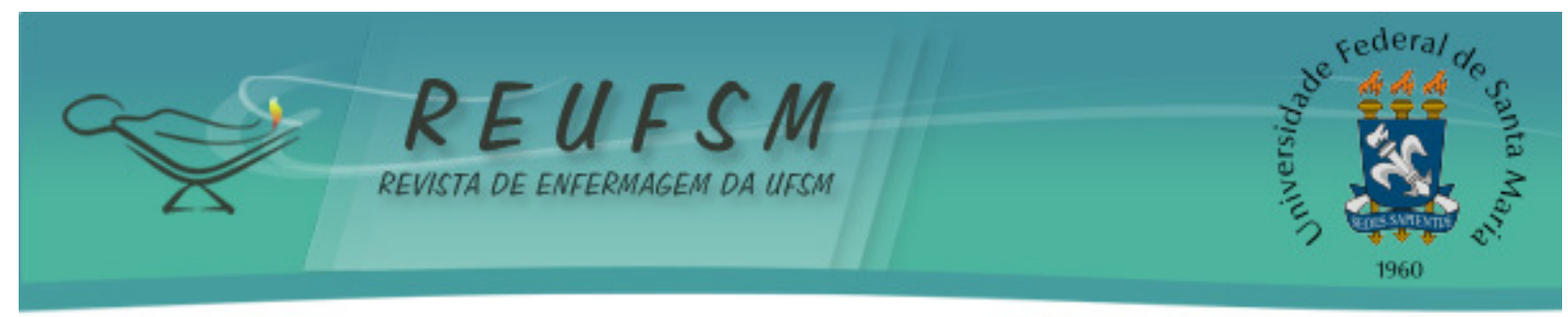

RELATO DE EXPERIÊNCIA

\title{
PARTICIPAÇÃO EM PROGRAMA DE INTERCÂMBIO INTERNACIONAL: CONTRIBUIÇÕES DA EXPERIÊNCIA DE GRADUAÇÃO-SANDUÍCHE EM ENFERMAGEM
}

\section{PARTICIPATION IN INTERNATIONAL EXCHANGE PROGRAM: CONTRIBUTIONS OF THE EXPERIENCE OF SANDWICH-UNDERGRADUATE STUDIES IN NURSING}

\section{PARTICIPACIÓN EN EL PROGRAMA DE INTERCAMBIO INTERNACIONAL: CONTRIBUCIÓNES DE LA EXPERIENCIA DE GRADO- SANDWICH EN ENFERMERÍA}

\author{
Renata de Moura Bubadué \\ Franco Carnevale ${ }^{2}$ \\ Cristiane Cardoso de Paula ${ }^{3}$ \\ Stela Maris de Mello Padoin ${ }^{4}$ \\ Eliane Tatsch Neves ${ }^{5}$
}

Doi: $10.5902 / 2179769291577922$

RESUMO: Objetivo: descrever a experiência de estudante de graduação de Enfermagem em Programa de intercâmbio internacional no Canadá. Método: 0 intercâmbio deu-se por meio de concessão de bolsa do Programa Ciência Sem Fronteiras do governo brasileiro, entre a Universidade Federal de Santa Maria/RS/Brasil e a McGill University, em Montreal/Quebec/Canadá. Foi desenvolvido no período de março a agosto de 2012, junto aos Programas de Graduação e de Pós-Graduação em diversos campos da saúde da criança e do adolescente. Resultados: atividades de inserção em grupo de pesquisa, disciplinas, cursos e projetos de pesquisa tiveram foco em cuidados paliativos, bioética e abordagem qualitativa de pesquisa. Considerações Finais: Essa vivência possibilitou o desenvolvimento acadêmico, pessoal e social bem como o início da tessitura de uma rede de pesquisa, contribuindo para o intercâmbio entre pesquisadores e o processo de internacionalização do Curso de Enfermagem. Descritores: Enfermagem; Intercâmbio educacional internacional; Programas de graduação em enfermagem.

ABSTRACT: Aim: to describe the experience of a Nursing Undergraduate student in an international exchange program in Canada. Methodo: The exchange happened through the awarding of a scholarship from the Brazilian government Science without Boarders Program, between the Federal University of Santa Maria/RS/Brazil and McGill University, in Montreal/Quebec/Canada. It was conducted from march to august 2012, with Graduate and Undergraduate Programs in diverse fields of child and adolescent health. Results: activities in research groups, courses, lectures and research projects focused on palliative care, bioethics and qualitative approaches in research. Final Considerations: This experience enabled academic, personal and social development, as well as the beginning

\footnotetext{
${ }^{1}$ Enfermeira graduada pela Universidade Federal de Santa Maria (UFSM/RS/Brasil), Mestranda em Enfermagem na Escola de Enfermagem Anna Nery da Universidade Federal do Rio de Janeiro (EEAN/UFRJ/RJ/Brasil). E-mail: renatabubadue@gmail.com

${ }^{2}$ Enfermeiro. PhD em Psicologia pela McGill University (Montreal/Canadá). Professor Titular da Escola de Enfermagem da Mcgill University. Orientador no Canadá. E-mail: franco.carnevale@mcgill.ca

${ }^{3}$ Enfermeira. Doutora em Enfermagem. Professora Adjunta da UFSM/RS/Brasil. Orientadora no Brasil. E-mail: cris_depaula1@hotmail.com

${ }^{4}$ Enfermeira. Doutora em Enfermagem. Professora Adjunta da UFSM/RS/Brasil. Coordenadora do Programa de Pós-Graduação em Enfermagem (PPGEnf) da UFSM/RS/Brasil. stelamaris_padoin@hotmail.com

${ }^{5}$ Enfermeira. Doutora em Enfermagem. Professora Adjunta da UFSM/RS/Brasil. Coordenadora do Curso de Graduação em Enfermagem da UFSM/RS/Brasil. E-mail: elianeves03@gmail.com
} 


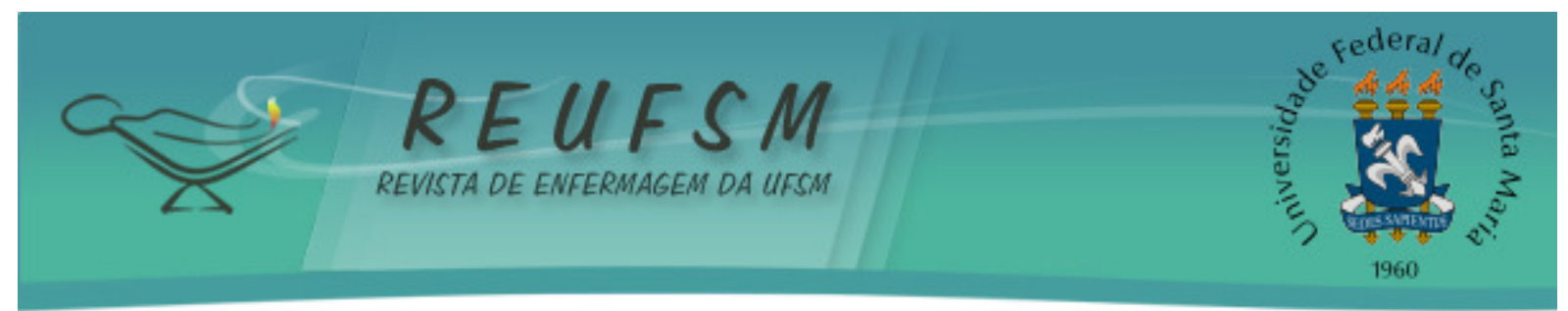

of constructing a research network, contributing to exchange among researchers and the process of internationalization of Nursing.

Descriptors: Nursing; International educational exchange; Education, nursing, diploma programs.

RESUMEN: Objetivo: describir la experiencia de estudiante de grado en enfermería en el Programa de intercambio internacional en Canadá. Método: El intercambio se llevó a cabo a través de la concesión de la subvención del Programa Ciencia Sin Fronteras del gobierno brasileño, entre la Universidad Federal de Santa Maria/RS/Brasil y la Universidad McGill de Montreal/Quebec /Canadá. Se desarrolló entre marzo a agosto de 2012, junto con los Programas de Grado y Postgrado en diversos campos de la salud del niño y del adolescente. Resultados: actividades de inserción en equipo de investigación, disciplinas, cursos e pesquisas se han centrado en los cuidados paliativos, la bioética y la investigación cualitativa. Consideraciones Finales: esta experiencia ha permitido el desarrollo académico, personal y social, así como el principio de tejer una red de investigación, lo que contribuye al intercambio entre los investigadores y el proceso de internacionalización del Curso de Enfermería.

Descriptores: Enfermería; Intercambio educacional internacional; Programas de graduación en enfermería

\section{INTRODUÇÃO}

O programa Ciência sem Fronteiras $(\mathrm{CsF})$ é resultado de uma iniciativa conjunta dos Ministérios da Ciência, Tecnologia e Inovação (MCTI) e do Ministério da Educação (MEC) do Brasil, organizado por meio de suas instituições de fomento: Conselho Nacional de Desenvolvimento Científico e Tecnológico (CNPq) e Coordenação de Aperfeiçoamento de Pessoal de Nível Superior (CAPES). ${ }^{1}$

O CSF consiste na concessão de bolsas a estudantes de graduação, pós-graduação e profissionais no exterior, a fim de propiciar a formação e capacitação de pessoas com elevado desenvolvimento acadêmico e profissional nas áreas de conhecimento definidas como prioritárias, sendo que a área de Biologia, Ciências Biomédicas e da Saúde está em terceiro lugar. ${ }^{1}$

Os objetivos do Programa são: investir na formação de pessoal altamente qualificado nas competências necessárias para o avanço da sociedade do conhecimento; aumentar a presença de pesquisadores e estudantes de diversos níveis em instituições de excelência no exterior; promover a inserção internacional das instituições brasileiras pela abertura de oportunidades semelhantes para cientistas e estudantes estrangeiros; ampliar o conhecimento inovador de pessoal das indústrias tecnológicas; e atrair jovens talentos científicos e investigadores altamente qualificados para trabalhar no Brasil. ${ }^{1}$

Com o CsF, o trânsito intercultural de estudantes de graduação aumentou de modo que, em um ano, 11.416 graduandos foram contemplados com bolsas de estudo. $\mathrm{Na}$ área de concentração "Biologia, Ciências Biomédicas e da Saúde", estão 9,11\% (1.041) dos bolsistas de graduação-sanduíche, sendo que apenas 3,55\% (37) são da Enfermagem. Os 37 estudantes contemplados têm atividades concluídas e em andamento, distribuídas na Europa, América do Norte e América do Sul. ${ }^{2}$

Há a predominância de estudantes na Europa com 25 bolsistas, sendo 13 na Itália, sete em Portugal, quatro no Reino Unido e um na Alemanha. Na América do Norte, estão dez estudantes, distribuídos entre Estados Unidos da América (oito) e Canadá (dois). 0 único país da América do Sul contemplado é o Chile com dois estudantes. ${ }^{2}$ No entanto, pode-se dizer que o fluxo de estudantes latino-americanos em intercâmbio ainda é baixo. ${ }^{3-4}$ 


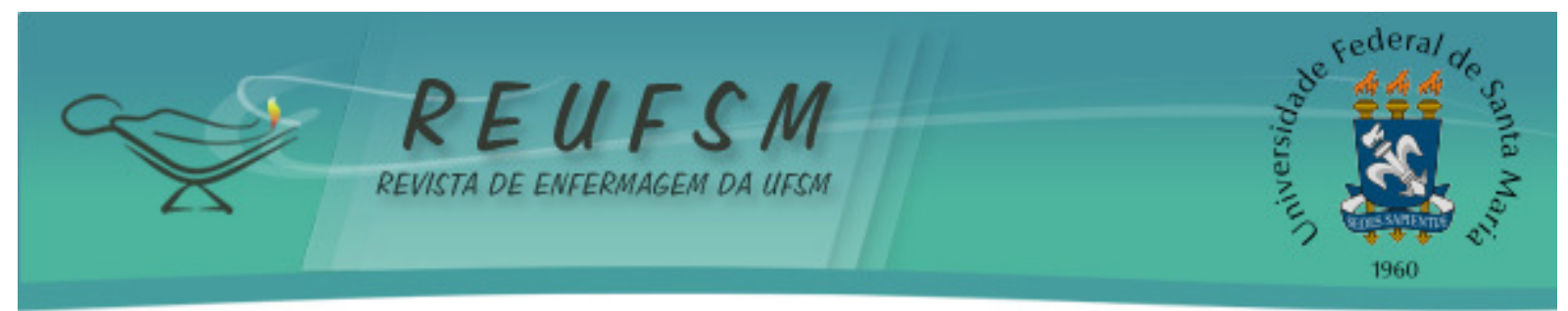

Os intercâmbios e mobilidades estudantis durante o período universitário contribuem para ampliar os horizontes do graduando no cenário exterior, por meio do contato com diversidades culturais. A interculturalidade é praticada durante a experiência no exterior por meio das relações estabelecidas com outros estudantes oriundos de diversos países. A internacionalização da graduação também aproxima os pesquisadores, os orientadores no Brasil e no exterior e os estudantes, especialmente aqueles de iniciação científica (IC), o que pode resultar em parcerias que beneficiam as universidades envolvidas e seus respectivos países. ${ }^{3-4}$

Diante da oportunidade deste intercâmbio e das vivências em atividades extracurriculares oferecidas no Departamento de Enfermagem da Universidade Federal de Santa Maria (UFSM/RS/BR) como a participação no grupo de pesquisa "Cuidado à Saúde das Pessoas, Família e Sociedade" (GP-PEFAS) e no Programa de Educação Tutorial do Curso de Enfermagem (PET Enfermagem) do Centro de Ciências da Saúde (CCS), optou-se pela participação no processo seletivo do Programa CsF que resultou em ser contemplada com a bolsa de graduação sanduíche no Canadá no final de 2011.

A inserção no GP-PEFAS do Curso de Enfermagem do CCS da UFSM em dezembro de 2008, proporcionou contato com a organicidade da ciência e multiplicidade da verdade no mundo da pesquisa, por meio da participação em projetos de pesquisa em temas relevantes relacionados à Saúde da Criança e do Adolescente. A organicidade, no sentido de mudança constante e a multiplicidade da verdade convergem com o paradigma construtivista em que as verdades transitam de acordo com a vivência/olhar de cada indivíduo, seja ele pesquisador ou sujeito de pesquisa. ${ }^{5}$

Destaca-se no GP-PEFAS, o Programa AIDS educação e cidadania que se insere na linha de pesquisa: "a vulnerabilidade e as demandas de cuidado de pessoas, famílias e sociedade no contexto da AIDS" e desenvolve estudos que buscam descrever a situação clínicoepidemiológica das pessoas que têm HIV/AIDS e compreender as vivências e as demandas de cuidado no seguimento ambulatorial de serviço especializado e na comunidade. ${ }^{6,7}$

A decisão pela vivência de graduação sanduíche no exterior ocorreu no sétimo semestre do Curso de Enfermagem quando a UFSM publicou o edital de bolsa do CNPq. A estudante já era bolsista de IC no grupo de GP-PEFAS há dois anos e meio e essa vivência impulsionou a curiosidade por novas experiências no âmbito da pesquisa. Assim, este trabalho tem como objetivo descrever a experiência de estudante de graduação de Enfermagem em Programa de intercâmbio internacional no Canadá.

\section{MÉTODO}

Relatar a experiência nesse programa requer descrever o primeiro desafio que foi a seleção institucional em que participaram estudantes de diferentes áreas de conhecimento. Essa foi dividida em quatro etapas que perpassavam a análise do currículo lattes, o índice de desenvolvimento acadêmico, a prova de conhecimento de língua estrangeira e a entrevista. Esse processo foi realizado em um intervalo de dois meses que culminou com a aprovação, em dezembro de 2011. Após a obtenção da bolsa de estudos, iniciaram-se os trâmites para o deslocamento e a instalação, que desencadearam sentimentos de entusiasmo e de temor frente à experiência de seis meses no exterior.

O cenário das atividades desenvolvidas no Canadá foi o Laboratório de pesquisa: Centre for Research on Children and Families, sob a orientação do professor Dr. Franco Carnevale na McGill University, localizada em Montreal/Quebec. Elaborou-se um plano de atividades em conjunto entre a graduanda, a orientadora no Brasil, o orientador no exterior e a Coordenação do Curso de Graduação em Enfermagem do CCS da UFSM. Este plano teve o intuito de 


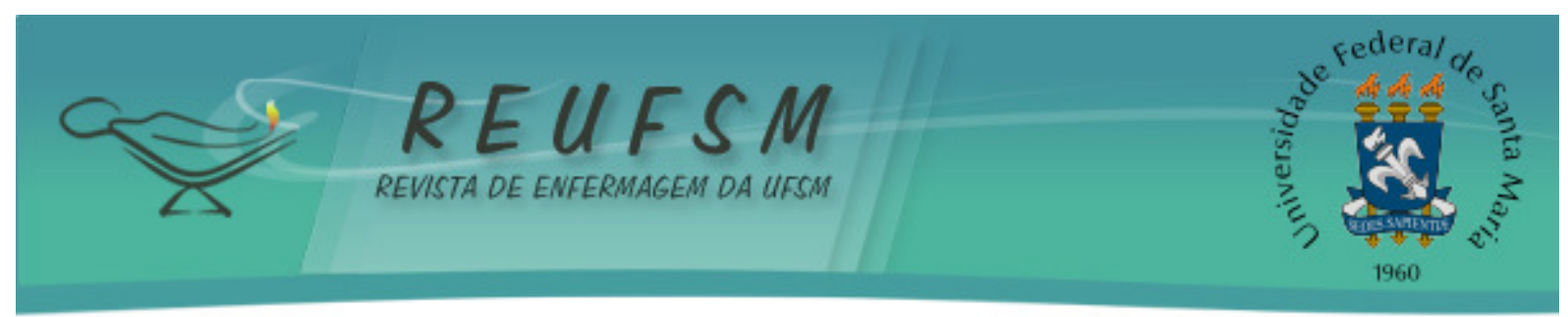

organizar as atividades de acordo com os interesses da graduanda e áreas de especialidade do orientador do exterior e foi desenvolvido no período de Março a Agosto de 2012.

\section{RESULTADOS}

Durante os seis meses que a estudante esteve na McGill University, foi possível a participação como ouvinte de cursos oferecidos na grade curricular da Graduação e PósGraduação, bem como atividades extracurriculares de pesquisas, palestras, visitas a centros de saúde e outras. Estas atividades proporcionaram a vivência com estudantes, docentes e profissionais de centros de referência do Canadá e de outros países. Isto contribuiu para o desenvolvimento pessoal e profissional, no sentido de ampliar a compreensão do significado de ser enfermeiro e das possibilidades de prevenção, promoção e assistência em saúde no contexto mundial.

Atividades em diversos campos da Saúde da Criança e do Adolescente, com predomínio dos focos de Cuidados Paliativos, Bioética e Pesquisa Qualitativa, foram eleitos como prioridade. Essa abordagem foi planejada em convergência com as vivências em campos de aula prática e de estágio curricular na Unidade de Internação Pediátrica do Hospital Universitário de Santa Maria (HUSM/RS/BR), de projetos de pesquisa e extensão vinculados ao GP-PEFAS e desenvolvidos no Serviço de Doenças Infectocontagiosas no Ambulatório Pediátrico ${ }^{6}$ e de projetos de extensão desenvolvidos pelo PET-Enfermagem. ${ }^{8}$

A solicitação de autorização para assistir aulas de graduação da disciplina Doenças Crônicas e Cuidados Paliativos foi motivada pela necessidade de ampliação das discussões do Projeto de Lei 524/09. Esse dispõe do direito das pessoas em fase terminal de doença e indica o direito à cuidados paliativos e mitigadores do sofrimento, proporcionais e adequados à situação, sem prejuízo de outros procedimentos terapêuticos que se mostrarem cabíveis. ${ }^{9}$ A disciplina oportunizou a leitura dos materiais oferecidos no curso, bem como a participação em palestras com profissionais e professores experientes na área. Assim, ampliou-se a reflexão crítica e o apreendizado de conceitos éticos e assistenciais importantes para a implementação dessa política pública no Brasil.

Complementar à essa disciplina que foi introdutória a de Cuidados Paliativos que são abordados no Canadá, proporcionou-se a participação em encontros do Pediatric Palliative Care Research Rounds no Montreal Children's Hospital (MCH). Esse grupo discute temas atuais referentes ao cuidado paliativo pediátrico por meio de apresentação de projetos de pesquisas, sejam finalizados ou em andamento, em um ambiente multidisciplinar. Isso possibilitou a interação com pesquisadores e profissionais, contribuindo para compreensão do cenário atual da pesquisa acerca dos cuidados paliativos em pediatria e possibilidades de desenvolvimento científico no Brasil.

Além disso, palestras e seminários sobre o assunto, promovidos pelo $\mathrm{MCH}$ ou pela universidade foram importantes. Também a possibilidade de encontrar com profissional músico-terapeuta, propiciou o conhecimento deste campo de trabalho e suas possibilidades terapêuticas para a criança no fim da vida.

Estas vivências despertaram a reflexão crítica da realidade política e prática brasileira que ainda é incipiente nos diálogos do processo de morte e morrer. ${ }^{10-11}$ Essas discussões são fundamentais para tornar o profissional enfermeiro apto a lidar com situações em que o paciente não se encontra em possibilidade de cura e proporcionar um cuidado especializado, humanizado e específico para as demandas de conforto no final da vida.

Nesse sentido, a bioética também se faz presente como instigante em discussões sobre decisões de fim da vida. No currículo de graduação da UFSM, é abordada de forma pontual, a fim de possibilitar a familiarização do estudante com o tema no campo prático de como deve ser sua postura profissional com o usuário. No entanto, as vivências em 


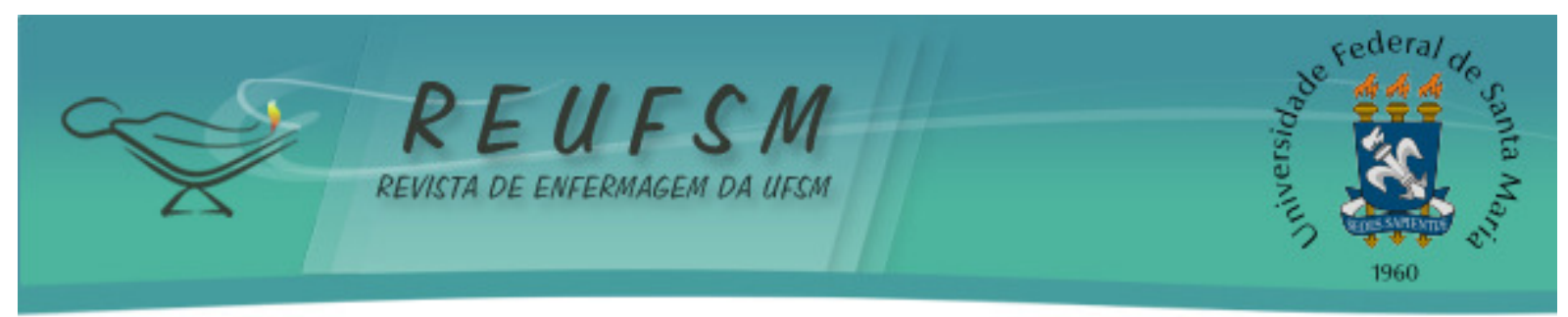

campos práticos na rede de atenção primária à saúde e hospitalar de Santa Maria/RS/BR, oportunizaram a elaboração de questionamentos pessoais quanto à postura profissional referente à voz do usuário, em especial da criança.

A participação no Curso de Ética em Prática Avançada oferecida pelo Programa de Mestrado em Enfermagem da McGill University, foi fundamental para a introdução de conceitos e conhecimentos que estão sendo aprofundados na participação em um projeto de pesquisa multidisciplinar e internacional, o qual tem como objetivos: identificar as atuais lacunas de conhecimento na ética pediátrica; identificar prioridades para o desenvolvimento científico e desenvolver um programa de pesquisa interdisciplinar que irá contemplar prioridades de pesquisa urgente e avançar os Estudos de Ética Pediátrica como um novo campo de investigação.

Essa pesquisa é coordenada pelo Dr. Franco Carnevale (School of Nursing, McGill) e inclui pesquisadores do Brasil, Canadá, Itália e França, em que a estudante está colaborando no subprojeto cujo objetivo é analisar normas éticas e legais em pediatria no Brasil $^{12}$ com pesquisadores da Universidade Federal do Rio de Janeiro (UFRJ/RJ/BR), da Universidade Estadual do Rio de Janeiro (UERJ/RJ/BR) e do Montreal Children's Hospital (McGill/Montreal/Canadá). Por meio desse projeto, emergiu a dimensão multidisciplinar da pesquisa e a possibilidade de contato com um campo pouco explorado no Brasil, despertando interesse e questionamentos para continuação de estudos nessa área.

A participação na Disciplina de Pesquisa Qualitativa do Programa de Doutorado em Enfermagem da McGill University desencadeou leituras e discussões aprofundadas referentes aos diferentes paradigmas e às principais metodologias nessa abordagem de pesquisa. A vivência com estudantes de doutorado e docentes deste Programa contribuiu na compreensão da dinâmica da pesquisa no Canadá e no mundo, bem como no desenvolvimento da habilidade de argumentação acerca das possibilidades metodológicas de uma pergunta de pesquisa. Além disso, estes conhecimentos foram compartilhados com pesquisadores brasileiros, contribuindo para a disciplina de Abordagem Qualitativa em Pesquisa na Saúde do Programa de Pós-Graduação em Enfermagem da UFSM.

Destaca-se que as experiências como estudante de IC, desde o início do Curso de Graduação em Enfermagem do CCS da UFSM, foram importantes para o aprofundamento em atividades referentes à metodologia o que foi fundamental para desenvolver a criticidade argumentativa acerca do uso de referenciais metodológicos como Fenomenologia, Etnografia, Teoria Fundamentada nos Dados, Etnografia Institucional e Descrição Interpretativa na investigação científica. Esse exercício foi realizado por meio da leitura do material que viabilizou a desconstrução e reconstrução de conceitos em discussões em sala de aula. Na integração do ensino e da pesquisa promovida pela IC, se estabelece uma relação de interdependência entre o sítio pedagógico e o científico. Isso permite a construção de um novo significado para a graduação, em que a sala de aula é vista como um espaço de construção de conhecimento. ${ }^{13-16}$

A graduação-sanduíche também possibilitou a conclusão da pesquisa intitulada: Vulnerabilidade ao Adoecimento de Crianças que têm HIV/AIDS em Transição da Infância para a Adolescência, referente ao Trabalho de Conclusão de Curso de Graduação (TCC), com orientadora no Brasil e orientador no exterior. A experiência internacional contribuiu com leituras e acesso a profissionais que trabalham no contexto de HIV/AIDS em Montreal, realizando visita à unidade em que as crianças e adolescentes soropositivos são atendidos e participam de um round no $\mathrm{MCH}$. Com o orientador no exterior e uma professora da Universidad de Antioquia (Colômbia), na época, visitante da McGill, foi possível discutir os resultados da pesquisa e fundamentar a discussão dos dados, com base no cenário do HIV na América Latina. Com isso, estabeleceu-se uma aliança científica para a possibilidade de desenvolvimento de atividades e intercâmbio cultural entre o Brasil, Colômbia e Canadá. 


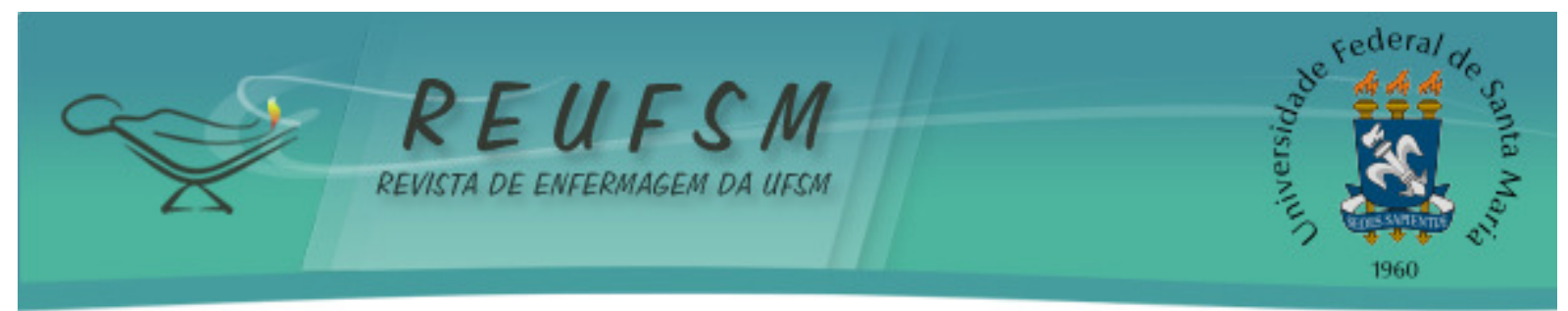

Ao retornar da graduação-sanduíche, foi instalada a defesa pública do TCC com banca examinadora na UFSM/RS/BR, composta por membros internos (orientadora no Brasil, coorientadora mestranda do PPGEnf e docentes do Curso de Graduação), externo (profissional do HUSM) e internacional (orientador no Canadá), o que possibilitou o aproveitamento de disciplina/créditos desenvolvidos no exterior e incluído em histórico acadêmico.

Dessa forma, a participação nesse programa de intercâmbio internacional contribuiu para o crescimento pessoal e acadêmico dos envolvidos. Por meio do contato com pesquisadores, profissionais e estudantes de diferentes países, pode-se vivenciar a Enfermagem no mundo acadêmico de uma importante universidade da América do Norte.

\section{CONSIDERAÇÕES FINAIS}

A imersão cultural no Canadá possibilitou o crescimento acadêmico, pessoal e social. A conquista de amizades, o exercício da língua inglesa, o contato com a língua francesa, a observação e adaptação de costumes em outro país e o desenvolvimento da independência e autonomia foram vivências importantes durante esse período.

Além disso, viabilizou-se entendimento da saúde em âmbito global e a familiarização do saber-fazer em Enfermagem na cultura canadense, bem como o modelo de atenção preconizado nos principais centros de referência. Destaca-se a importância e a realidade do trabalho multidisciplinar, tendo o enfermeiro como agente protagonista e autônomo no processo do planejamento do cuidado.

Durante esse período, iniciou-se a tessitura de uma rede de pesquisa, a fim de promover $o$ intercâmbio de conhecimentos e a possibilidade do desenvolvimento de investigações interinstitucionais, contribuindo para a internacionalização do curso de Enfermagem da UFSM.

Convergente com os objetivos do CsF houve o investimento no desenvolvimento técnico-científico para contribuir com o avanço e visibilidade da Enfermagem brasileira. Isso se deu por meio da construção de artigos encaminhados para publicação, de trabalhos apresentados em eventos da área e da divulgação da possibilidade de intercâmbio durante a graduação na mídia local.

Ressalta-se que foram essenciais para a realização do intercâmbio internacional o incentivo e apoio da família, da orientadora no Brasil, do GP-PEFAS, da Coordenação do Curso de Graduação em Enfermagem e da Secretaria de Apoio Internacional da UFSM. E, sem dúvida, a solicitude do orientador na McGill University, professor Dr. Franco Carnevale, foi fundamental para o sucesso desta experiência.

O compartilhamento dessa experiência tem o propósito de instigar estudantes de graduação em Enfermagem e de outras áreas a participarem da seleção do CsF como uma oportunidade de mobilidade acadêmica internacional, assim como o interesse e a possibilidade de repetir a experiência durante a pós-graduação.

\section{Agradecimentos}

Ao Conselho Nacional de Desenvolvimento Científico e Tecnológico (CNPq) pela bolsa de estudos concedida à estudante de Enfermagem.

\section{REFERÊNCIAS}

1. Presidência da República (BR). Decreto $n^{\circ} 7.642$, de 13 de dezembro de 2011. Institui o Programa Ciência sem Fronteiras [Internet].[acesso em 2012 nov 15]. Disponível em: http://www.planalto.gov.br/ccivil_03/_Ato2011-2014/2011/Decreto/D7642.htm. 


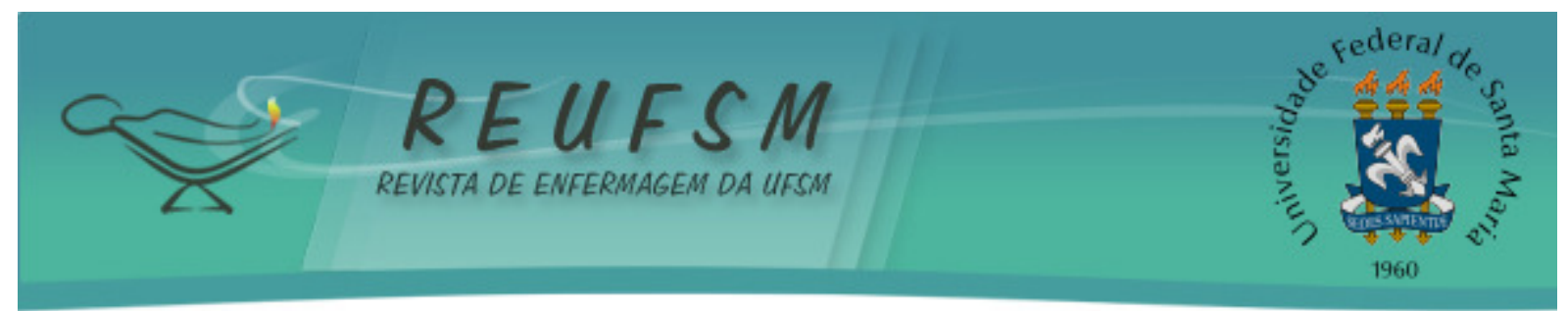

2. Ministério da Ciência, Tecnologia e Inovação/CNPq, Ministério da Educação/MEC. Ciência sem Fronteiras. Bolsistas pelo mundo [Internet]. [acesso em 2012 nov 14]. Disponível em: http://www.cienciasemfronteiras.gov.br/web/csf/bolsistas-pelo-mundo.

3. Souza Junior JM. A internacionalização e a mobilidade na Educação Superior: o debate na América Latina. Rev Iniciac Cient FFC. 2010;10(2).

4. Nogueira MA, Aguiar AMS, Ramos VCC. Fronteiras desafiadas: a internacionalização das experiências escolares. Educ Soc (Campinas). 2008;29(103):355-76.

5. Guba EG, Lincoln YS. Competing paradigms in qualitative research. In: Denzin NK, Lincoln YS, eds. Handbook of qualitative research. Sage Publications; 1993. p. 105-17.

6. Padoin SMM, Paula CC. Programa AIDS, educação e cidadania: perspectivas para a segunda década de extensão. Saúde (Santa Maria) [Internet]. 2012 [acesso em 2012 nov 14];38(1):83-92. Disponível em: http://cascavel.ufsm.br/revistas/ojs2.2.2/index.php/revistasaude/article/view/3796/pdf.

7. Padoin SMM, Paula CC, Hoffmann IC, Valadão MC, Rodrigues AP, Langendorf TF. Alimentação de crianças que convivem com Aids: vivências de familiares/cuidadores em atividade grupal. Rev Enferm UFSM [Internet]. 2012 jan/abr [acesso em 2012 nov 21];2(1):213-21. Disponível em: http://cascavel.ufsm.br/revistas/ojs2.2.2/index.php/reufsm/article/view/2804/3146.

8. Ressel LB, Silva SC, Santos CC, Frescura CJ, Stumm KE, Ribeiro DB, et al. A vivência de acadêmicos de enfermagem como oficineiros em grupos de adolescentes. REME Rev Min Enferm. 2011;15(2):290-5.

9. Senado Federal (BR). Projeto de Lei $n^{\circ}$ 524, de 25 de novembro de 2009. Dispõe do direito das pessoas em fase terminal de doença. Brasília; 2009.

10. Silva Júnior FJ, Santos LCS, Moura PVS, Melo BMS, Monteiro CFS. Processo de morte e morrer: evidências da literatura científica de enfermagem. Rev Bras Enferm [Internet]. 2011 [acesso em 2012 nov 20];64(6):1122-6. Disponível em: http://www.scielo.br/scielo.php?script=sci_arttext\&pid=S0034-71672011000600020.

11. Pessalacia JDR, Oliveira VC, Rennó HMS, Guimarães EAA. Perspectivas do ensino de bioética na graduação em enfermagem. Rev Bras Enferm [Internet]. 2011 [acesso em 2012 nov 14];64(2):393-8.

Disponível

em:

http://www.scielo.br/pdf/reben/v64n2/a29v64n2.pdf.

12. Carnevale F. Ethical considerations in pediatric nursing. Rev Soc Bras Enferm Ped [Internet]. 2012 [acesso em 2012 nov 14];12(1). Disponível em: http://www.sobep.org.br/revista/component/zine/article/152-ethical-considerations-inpediatric-nursing.html.

13. Massi L, Queiroz SL. Estudos sobre iniciação científica no Brasil: uma revisão. Cad Pesqui [Internet]. 2010 [acesso em 2012 nov 21];40(139):173-97. Disponível em: http://www.scielo.br/pdf/cp/v40n139/v40n139a09.pdf.

14. Uesugui HM, Chiaratto RA, Fagundes DS, Zorzi AC, Framil GB, Souza JM. Iniciação científica em Enfermagem: instrumento de transformação do saber. Rev Faema [Internet]. 2011 [acesso em 2012 nov 20];2(1):86-92. Disponível em: http://www.faema.edu.br/revistas/index.php/Revista-FAEMA/article/view/41

15. Neuenfeldt DJ, Schuck RJ, Munhoz A, Mittelstadt J, Miorando TM, Rochenback R. Iniciação à pesquisa no ensino superior: desafios dos docentes no ensino dos primeiros 


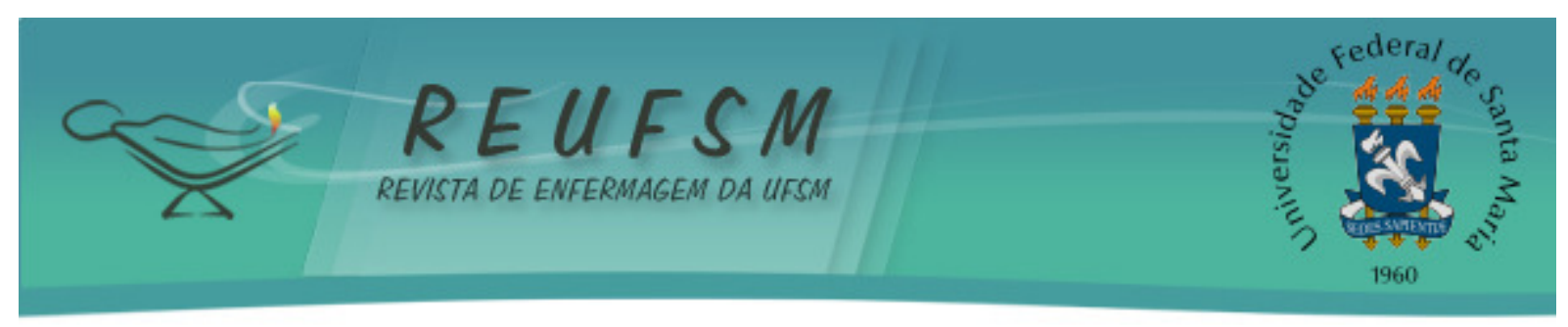

passos. Ciênc Educ (Bauru) [Internet]. 2011 [acesso em 2012 nov 17];17(2):289-300. Disponível em: http://www.scielo.br/pdf/ciedu/v17n2/a03v17n2.pdf.

16. Novais NN, Silva LWS. O ambiente da investigação em enfermagem: um relato de experiência. Rev Bras Enferm [Internet]. 2011 [acesso em 2012 nov 23],64(3):605-10. Disponível em: http://www.scielo.br/pdf/reben/v64n3/v64n3a29.pdf.

Data de recebimento: 19/02/2013

Data de aceite: $12 / 12 / 2013$

Contato com autor responsável: Renata de Moura Bubadué Endereço postal: Universidade Federal do Rio de Janeiro, Escola de Enfermagem Anna Nery, Rua Afonso Cavalcanti, 275. Cidade Nova. CEP 20211-110. Rio de Janeiro, RJ. E-mail: renatabubadue@gmail.com 